\title{
Proteins and medium-flow conditions: how they influence the degradation of magnesium
}

Ruiqing Hou PhD

Researcher, Institute of Advanced Wear and Corrosion Resistant and Functional Materials, Jinan University, Guangzhou, China (Orcid:0000-0001-6240-5239)

Frank Feyerabend PhD

Researcher, Metallic Biomaterials Division, Institute of Materials Research, Helmholtz-Zentrum Geesthacht, Geesthacht, Germany

(Orcid:0000-0001-9532-0207)
Regine Willumeit-Römer PhD

Professor, Metallic Biomaterials Division, Institute of Material Research, Helmholtz-Zentrum Geesthacht, Geesthacht, Germany (corresponding author: regine.willumeit@hzg.de) (Orcid:0000-0002-6004-6946)

To understand the magnesium (Mg) degradation mechanism in vivo, a variety of in vitro investigations have been conducted. However, there is still a gap between in vitro results and in vivo observations. Herein, the combined effect of proteins and medium-flow conditions on magnesium degradation was studied. Bovine serum albumin (BSA) and fetal bovine serum (FBS) were employed as the commonly used protein components for in vitro tests. Static, medium-changing (semi-static) and continuous-medium-flow (dynamic) conditions were used as different conditions. The results showed the highest inhibition efficiency of BSA and FBS on magnesium degradation under static conditions, while the efficiency largely decreased under semi-static or dynamic conditions, particularly for BSA. The possible explanations are related to the composition of degradation products and the integrity of the degradation layer. These results emphasize the importance of considering effects from different factors during magnesium degradation.

\section{Notation \\ A surface area exposed to the medium \\ $\mathrm{DR}_{\text {with protein }}$ degradation rate of magnesium $(\mathrm{Mg})$ in the protein-containing medium \\ $\mathrm{DR}_{\text {without protein }}$ degradation rate of magnesium in the medium without protein \\ $m_{1} \quad$ weight of the sample before immersion \\ $m_{2} \quad$ weight of the sample after the removal of degradation products \\ $\begin{array}{ll}t & \text { immersion time } \\ \rho & \text { density of pure magnesium }\end{array}$}

\section{Introduction}

Magnesium $(\mathrm{Mg})$ and its alloys have gained increasing attention as metallic biomaterials due to their good biodegradability and compatibility. ${ }^{1-3}$ However, there is still a large gap between the in vitro and in vivo results for magnesium degradation due to the complex environment under in vivo conditions. ${ }^{46}$ To fill this gap and lay a solid foundation for the design and development of magnesium-based materials, it is necessary to gain a deeper understanding of the in vivo degradation mechanism of magnesiumbased biomaterials. This, however, is quite difficult because the degradation in vivo cannot be monitored at all time points and with high spatial, chemical and temporal resolution. Thus, how parameters, which play most likely a role in in vivo degradation, influence the process should be systematically investigated.

To achieve this goal, degradation in different solutions has been studied: from simple salt solutions to more complex cell culture media. $^{7-9}$ The effects of various physiological parameters, such as buffering, ${ }^{10,11}$ medium inorganic composition, ${ }^{10,12}$ organic molecules ${ }^{13-16}$ and stress loading, ${ }^{17,18}$ have been investigated. In comparison with the results obtained under in vivo conditions,
Earle's balanced salt solution and simulated body fluid have been recommended as proper testing solutions for magnesium degradation in terms of degradation rate. ${ }^{7,8}$ However, organic molecules in the medium have been found to promote the formation of calcium phosphate $\left(\mathrm{Ca}_{3}\left(\mathrm{PO}_{4}\right)_{2}\right)$ on the magnesium surface and modify the composition of degradation products. ${ }^{14,19}$ These degradation products play an important role in some biological processes - for instance, cell adhesion and surface biomineralization. ${ }^{20}$ In sum, it is obvious that very complex chemical and biological reactions take place during the degradation in vivo, but the understanding about the process is still not sufficient.

Among organic molecules, proteins are the most abundant and the major organic components in human plasma. Their influence on magnesium degradation represented by bovine serum albumin (BSA) and fetal bovine serum (FBS) has been widely studied in different solutions under different conditions. ${ }^{21-23}$ In general, their influence is mainly ascribed to protein adsorption on the magnesium surface and/ or the binding/chelating to released ions. ${ }^{15,24,25}$ The adsorption of proteins can decrease the degradation rate of magnesium due to the barrier effect of the adsorbed protein film, while the binding/chelating effect of proteins results in the accelerated degradation of magnesium because of the interference with the formation of degradation products, which leads to an alloy- and medium-dependent influence of proteins on magnesium degradation. ${ }^{24,26}$

Another factor that influences degradation is the availability of fresh medium to avoid saturation effects. This is realized in the body by constant transport phenomena but often not considered in in vitro experiments. To realize the dynamic metabolic equilibrium in the human body, different testing methods for degradable magnesium have been developed, such as simple static immersion in a certain solution volume during the whole testing period, partially or entirely 
changing the solution after certain immersion intervals (semistatic) $)^{8,19,27}$ and more complex medium-flow conditions by way of a bioreactor system (dynamic). ${ }^{28,29}$ However, the synergistic effect of different parameters on magnesium degradation so far has not achieved enough attention - for example, the comprehensive influence of proteins and the medium-flow conditions.

Therefore, in the present study, the interplay between proteins (BSA and FBS) and three different conditions (static, semi-static and dynamic conditions) on magnesium degradation was investigated.

\section{Materials and methods}

\subsection{Materials}

The pure magnesium used in this study was cut into small disks with dimensions of $10 \mathrm{~mm} \times 10 \mathrm{~mm} \times 4 \mathrm{~mm}$ from a cast magnesium ingot (purity: 99.94 wt.\%, Magnesium Elektron, Manchester, UK), the detailed composition and the microstructure of the material have been presented in a previous report. ${ }^{13}$ Before the immersion test, the surface of each of the pure magnesium samples was ground successively using 800, 1200 and 1500 grid silicon carbide $(\mathrm{SiC})$ abrasive paper (Schmitz-Metallographie $\mathrm{GmbH}$, Herzogenrath, Germany) and then ultrasonically cleaned in $n$-hexane $\left(\mathrm{C}_{6} \mathrm{H}_{14}\right)$, acetone $\left(\mathrm{C}_{3} \mathrm{H}_{6} \mathrm{O}\right)$ and $100 \%$ ethanol $\left(\mathrm{C}_{2} \mathrm{H}_{5} \mathrm{OH}\right)$ (Merck KGaA, Darmstadt, Germany) for $20 \mathrm{~min}$. To avoid contamination and maintain sterilization, the samples were ultrasonically immersed in $70 \%$ ethanol for $20 \mathrm{~min}$ and finally dried under sterilized conditions prior to use.

\subsection{Immersion tests}

The cell culture medium Dulbecco's modified Eagle medium GlutaMax-I (DMEM) (Life Technologies, Darmstadt, Germany) was used as the base medium for the immersion tests due to the similar degradation of magnesium in it to that under in vivo conditions. $^{19}$ To prepare the protein-containing medium, $3.4 \mathrm{mg} / \mathrm{ml} \mathrm{BSA}$ and $10 \%$ FBS were added in DMEM, since they have similar protein concentration. ${ }^{19}$ Thus, three different media, DMEM, DMEM $+3 \cdot 4 \mathrm{mg} / \mathrm{ml}$ BSA and DMEM $+10 \%$ FBS, were used for the immersion tests.

Static, semi-static and dynamic conditions (Figure 1) were the different medium-flow conditions used for the immersion test under cell culture conditions $\left(37^{\circ} \mathrm{C}, 5 \%\right.$ carbon dioxide $\left(\mathrm{CO}_{2}\right), 20 \%$ oxygen $\left(\mathrm{O}_{2}\right), 95 \%$ relative humidity). Each sample was immersed in $20 \mathrm{ml}$ medium for $14 \mathrm{~d}$ of immersion; thus, the ratio of the medium volume to the sample surface $(\mathrm{V} / \mathrm{S})$ was around $5 \cdot 88$, which refers to the static condition. For the semi-static condition, $3.5 \mathrm{ml}$ medium was used for each sample $(\mathrm{V} / \mathrm{S}=1.03)$ and the medium was refreshed after 3, 5, 7, 10 and $12 \mathrm{~d}$ of immersion. Therefore, the volume of the medium used for each sample was $21 \mathrm{ml}$ for the semi-static condition. To present a medium-flow condition, a bioreactor was supplied with $20 \mathrm{ml}$ unrenewable medium per sample (V/S $=5 \cdot 88)$ during the test at a medium flow rate of $2 \mathrm{ml} / \mathrm{min}$ in the tube (dynamic condition). After certain immersion times under different conditions, the $\mathrm{pH}$ (Sentron Argus $\mathrm{X} \mathrm{pH}$ meter, Fisher Scientific, Schwerte, Germany) and osmolality (Osmomat 030, Gonotec, Berlin, Germany) of the medium were measured. For each condition or medium, four replicates were used; three of them were used to calculate the degradation rate of samples by way of weight loss measurement, and the one was used to characterize the surface degradation products.

\subsection{Degradation rate}

The degradation rates of magnesium under different conditions were determined by weight-loss measurement. The weight of each sample ( $m_{1}$ in grams) was determined before immersion. After $14 \mathrm{~d}$ of immersion, the degradation products on the magnesium surface were removed by immersing in $180 \mathrm{~g} / \mathrm{l}$ chromium trioxide $\left(\mathrm{CrO}_{3}\right)$ in distilled water (VWR International, Darmstadt, Germany) for $20 \mathrm{~min}$. Subsequently, the sample was cleaned with distilled water and ethanol (Merck KGaA, Darmstadt, Germany). After drying, the weight of the sample was determined again $\left(m_{2}\right.$ in grams). The degradation rate of magnesium (DR in millimeters/ year) can be calculated by using the following equation

$$
\text { 1. } \mathrm{DR}=\frac{8.76 \times 10^{4}\left(m_{1}-m_{2}\right)}{A \rho t}
$$

where $A$ is the surface area exposed to the medium $\left(\mathrm{cm}^{2}\right) ; \rho$ is the density of pure magnesium $\left(1.74 \mathrm{~g} / \mathrm{cm}^{3}\right)$; and $t$ is the immersion time (h). Furthermore, the inhibition efficiency (IE in percent) of proteins to the degradation of magnesium can be calculated as follows
Static condition

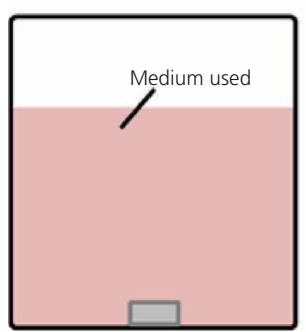

Semi-static condition

Every 2 or $3 \mathrm{~d}$

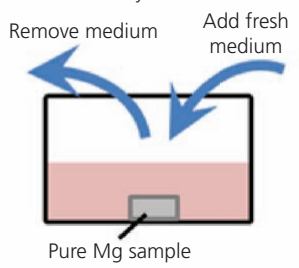

Dynamic condition

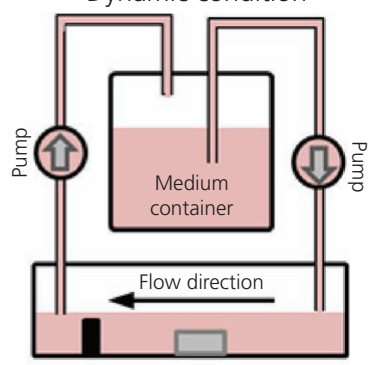

Figure 1. Schematic illustration of the three different immersion conditions used 


$$
\text { 2. } \mathrm{IE}=\frac{\mathrm{DR}_{\text {without protein }}-\mathrm{DR}_{\text {with protein }}}{\mathrm{DR}_{\text {without protein }}} \times 100 \%
$$

Herein, $\mathrm{DR}_{\mathrm{without}}$ protein is the degradation rate of magnesium in the medium without proteins (DMEM) and $\mathrm{DR}_{\text {with protein }}$ is the degradation rate of magnesium in the protein-containing medium (DMEM + BSA or DMEM + FBS).

\subsection{Surface characterization}

After immersion, sample surfaces were analyzed by scanning electron microscopy (SEM; Phenom-World, Eindhoven, the Netherlands) at $15 \mathrm{kV}$ acceleration voltage in backscattering mode. The composition of surface degradation products was identified by point analysis of X-ray energy-dispersive spectroscopy (EDS). At least five points were analyzed for the composition of surface products. Infrared (IR) spectra of the sample surface were acquired by taking 512 scans at a resolution of $2 \mathrm{~cm}^{-1}$ in reflectance mode (Bruker Hyperion 2000, Ettlingen, Germany). The Bruker Opus version 7.5.18 software was used to analyze the IR results.

The samples after immersion were embedded in resin to prepare cross-sections of the degradation layer. After successive grinding with 800,1500 and 2400 grid abrasive paper and polishing with colloidal silica $\left(\mathrm{SiO}_{2}\right)$ suspension (Cloeren Technology $\mathrm{GmbH}$, Wegberg, Germany), the thickness of the degradation layer was evaluated from SEM images of the cross-section (Tescan Vega3 SB, Brno, Czech Republic). The distribution of elements in the degradation layer was further analyzed by EDS mapping, which was performed at $15 \mathrm{kV}$ acceleration voltage at a resolution of 256 pixels. The acquiring time was $80 \mathrm{~ms}$ per pixel. The thickness of the degradation layer was taken from the cross-sectional SEM images with at least 80 points for each condition.

\subsection{Statistical analysis}

Statistical analysis of the degradation rate and layer thickness was carried out by using one-way analysis of variance on ranks with Dunn's multiple-comparison post hoc tests. Figures 1, 5 and 7 were organized in the Adobe Photoshop CS5 software (Adobe Systems Incorporated, San Jose, USA); the others were created in the OriginPro 8 software (OriginLab Corporation, Wellesley Hills, USA).

\section{Results}

\subsection{Degradation rate, $\mathrm{pH}$ and osmolality}

Figure 2 shows the degradation rate of pure magnesium in DMEM, DMEM + BSA and DMEM + FBS under different conditions. The results revealed that the highest degradation rate of pure magnesium was in DMEM under static immersion $(1.358 \pm 0.068 \mathrm{~mm} /$ year). Under this condition, the addition of BSA and FBS significantly reduced the degradation rate of pure magnesium to $0.444 \pm 0.052$ and $0.188 \pm 0.001 \mathrm{~mm} /$ year, respectively. Obviously, the semi-static condition $(0 \cdot 301 \pm$

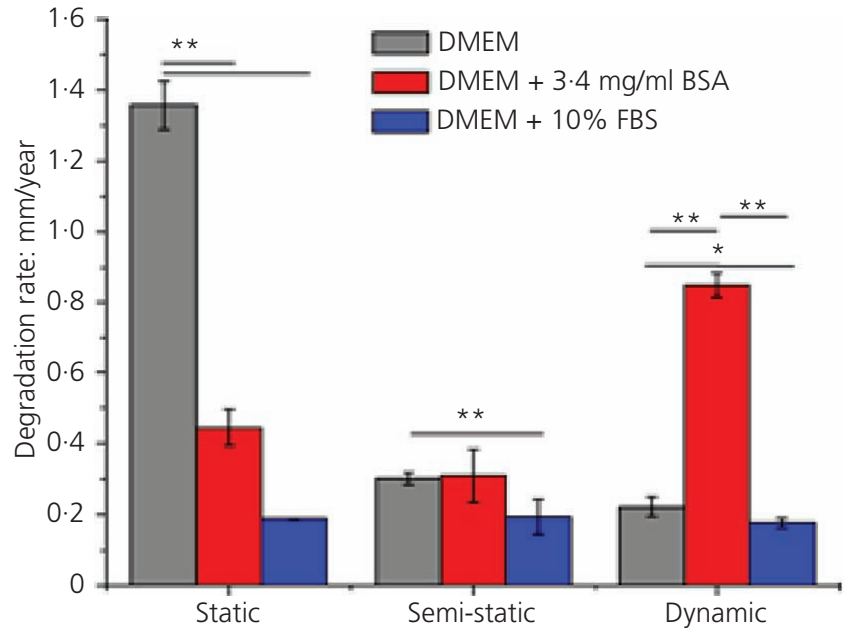

Figure 2. Degradation rate of pure magnesium in the DMEMbased medium under different conditions. Significance level: ${ }^{*} p<0 \cdot 05,{ }^{* *} p<0.01$

$0.017 \mathrm{~mm} /$ year $)$ and dynamic condition $(0.222 \pm 0.028 \mathrm{~mm} /$ year $)$ also presented lower degradation rates for pure magnesium in DMEM than the static condition. The presence of BSA under the semi-static condition resulted in a degradation rate for pure magnesium comparable with that in DMEM, while it led to a much faster degradation $(0.849 \pm 0.035 \mathrm{~mm} /$ year $)$ under the dynamic condition. More importantly, the addition of FBS always decreased the degradation rate and a relatively stable degradation rate $(\sim 0 \cdot 180 \mathrm{~mm} /$ year $)$ was determined in DMEM $+10 \%$ FBS irrespective of which condition was used.

Furthermore, the inhibition efficiency of proteins on magnesium degradation under different conditions was calculated based on Equation 2, as shown in Figure 3. The figure shows the higher inhibition efficiency of FBS than that of BSA regardless of the

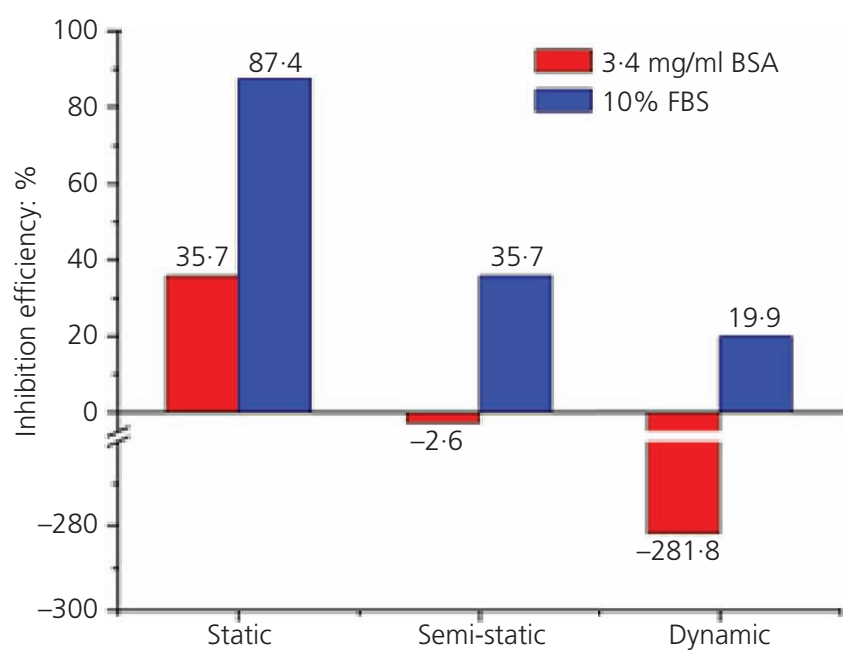

Figure 3. Inhibition efficiency of proteins (BSA or FBS) on magnesium degradation 
used condition. Moreover, positive values were determined for FBS irrespective of conditions, while BSA presented negative values for semi-static and dynamic conditions, indicating that FBS was more efficient than BSA for preventing magnesium degradation. However, the most conspicuous observation is that the inhibition efficiency for both BSA and FBS decreased in the following order: static condition $>$ semi-static condition $>$ dynamic condition. This suggests that the medium-flow condition can affect the interaction between proteins and the magnesium surface.

The variations in the $\mathrm{pH}$ and osmolality of the medium during immersion under different conditions are shown in Figure 4. The results showed that the $\mathrm{pH}$ was always lower than $8 \cdot 5$, indicating the good buffering capacity of the carbon dioxide/bicarbonate $\left(\mathrm{HCO}_{3}{ }^{-}\right)$system used in this study. Under the static condition, both the $\mathrm{pH}$ and osmolality of the medium gradually increased, but the fastest increase was observed in DMEM, showing a good agreement with the degradation rate (Figure 2). For the semi-static condition, the $\mathrm{pH}$ first showed a high value and then gradually decreased or remained stable. A similar variation could be found for osmolality. This was caused by the low volume of the medium used, the refreshing of the medium every 2 or $3 \mathrm{~d}$ and the decreasing degradation rate of magnesium with the immersion time. ${ }^{30}$ In the case of the dynamic condition, the variations in $\mathrm{pH}$ and osmolality were similar to that under the static condition, but with a relatively fast increase for osmolality, particularly for the initial several days, suggesting the fast diffusion process under the dynamic condition.

\subsection{Top-view degradation morphology and degradation products}

The top-view surface morphologies of pure magnesium in different media under different conditions are shown in Figure 5. The cracks during the drying process are visible for all samples. Some holes were observed on the degradation product layers formed in DMEM under the static condition, which was caused by the peeling off of degradation products due to hydrogen $\left(\mathrm{H}_{2}\right)$ evolution during immersion. Except for the defects existing in the degradation product layer (indicated by the arrows in Figure 5) formed in DMEM under static immersion and in DMEM + BSA under static and dynamic immersion, few differences were found.

EDS was performed to characterize the composition of degradation products on pure magnesium, as listed in Table 1 . In general, the products were composed of magnesium, oxygen $(\mathrm{O})$, phosphorus $(\mathrm{P})$, calcium $(\mathrm{Ca})$, carbon $(\mathrm{C})$ and sodium $(\mathrm{Na})$. The existence of sodium was caused by the remained composition of medium after immersion. Oxygen and magnesium are the major elements of the degradation products due to the formation of magnesium-based products, such as magnesium hydroxide $(\mathrm{Mg}$ $\left.(\mathrm{OH})_{2}\right)$ and magnesium carbonate $\left(\mathrm{MgCO}_{3}\right){ }^{31}$ The presence of calcium resulted from the formation of calcium-involved insoluble products, which were derived from calcium ions $\left(\mathrm{Ca}^{2+}\right)$ in the testing medium. The precentages of phosphorus and calcium in degradation products were evidently different, which generally presented higher contents in medium containing BSA or FBS, except under static condition. This is in accordance with the
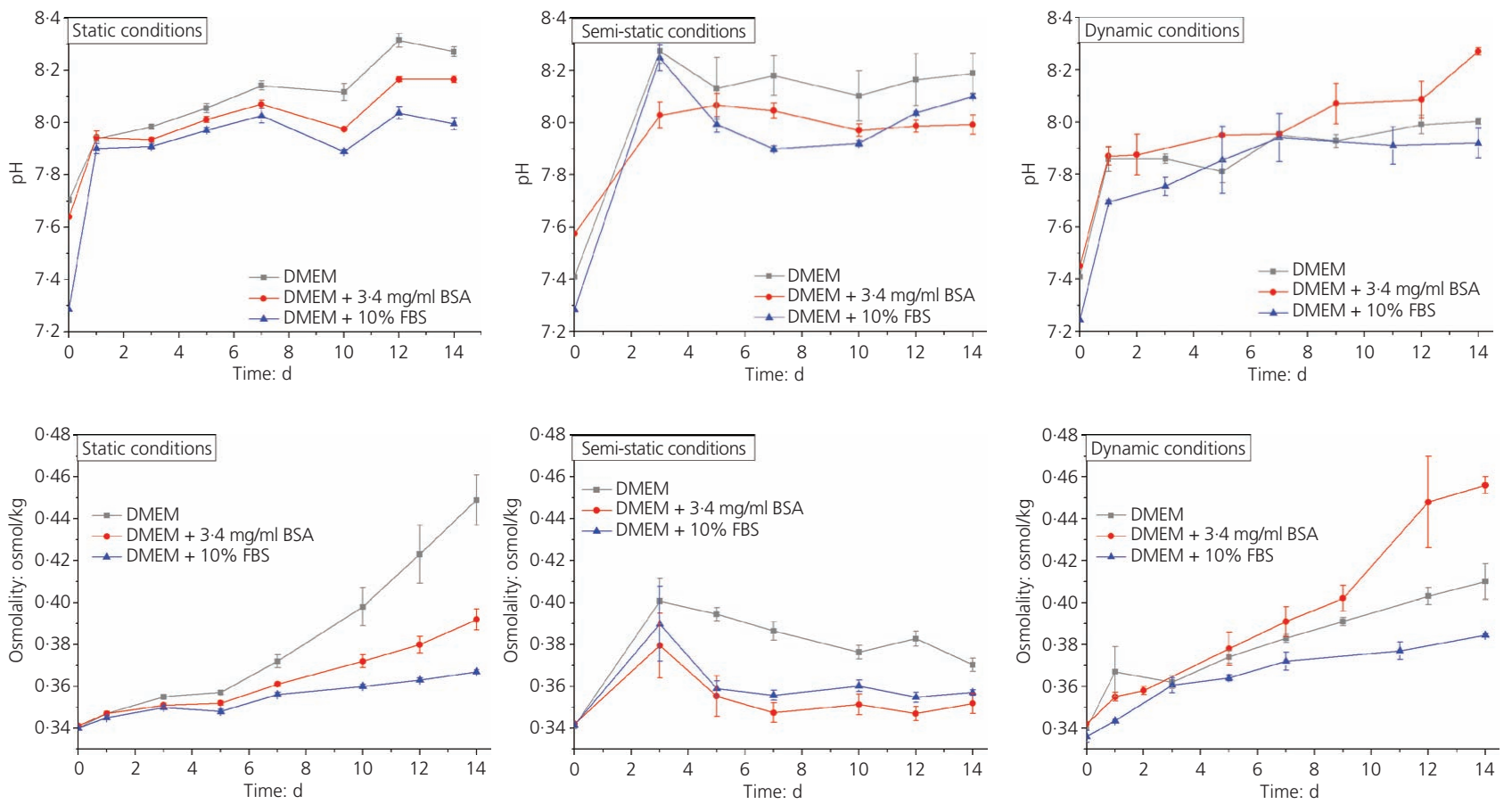

Figure 4. Variations in $\mathrm{pH}$ and osmolality during immersion in different media under different conditions 

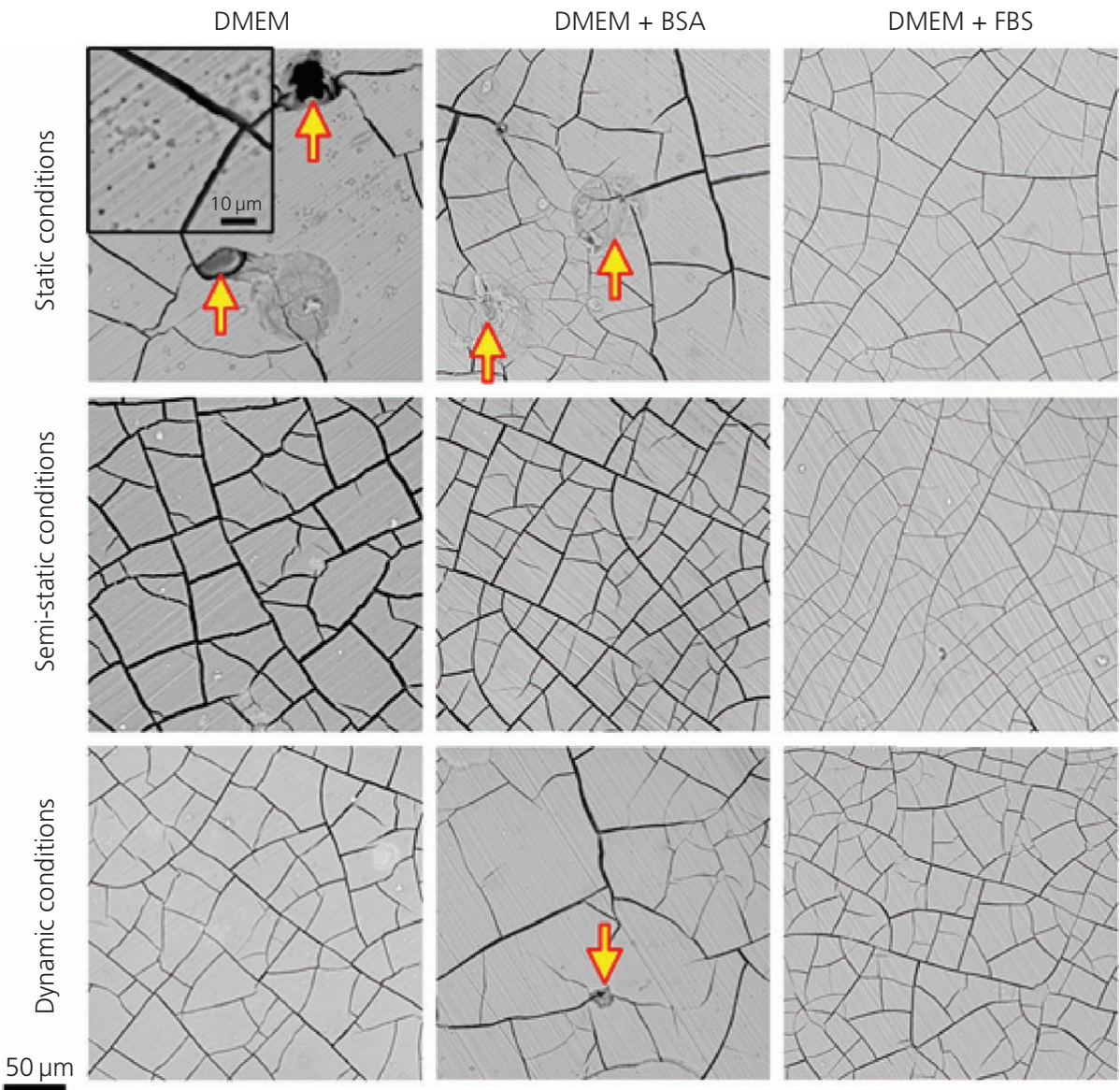

Figure 5. Top-view SEM images of samples after immersion in different media under different conditions (the arrows point to the defects of the degradation product film on samples)

authors' previous report ${ }^{13,19}$ that organic molecules promote the formation of calcium-phosphorus salts on the magnesium surface under cell culture conditions.

The degradation products were further characterized by using IR spectra. The typical spectra are shown in Figure 6. The very broad band in the range $3600-2700 \mathrm{~cm}^{-1}$ is caused by water, which always exists on hydrophilic surfaces due to the strong hydrogen (H) bonds of water. The band around $1640 \mathrm{~cm}^{-1}$ is ascribed to the $\mathrm{OH}$ bending of absorbed water and/or the amide I of organic molecules absorbed on the magnesium surface. ${ }^{19,32}$ The broad band from 1600 to $1300 \mathrm{~cm}^{-1}$ should be a result of the stretching of the carboxyl bond in organic molecules and carbonates. ${ }^{23,33}$ The following broad bond at $1025 \mathrm{~cm}^{-1}$ is attributed to the stretching of the phosphate $\left(\mathrm{PO}_{4}{ }^{3-}\right)$ bond, ${ }^{34}$ and the bending of the carbonate bond resulted in the bonds at 859 and $579 \mathrm{~cm}^{-1} .{ }^{13}$ The weak band near $3700 \mathrm{~cm}^{-1}$ is an indication of the existence of magnesium hydroxide. ${ }^{35}$ Similar products on the magnesium surface in different media can be concluded as carbonates, phosphates, adsorbed organic molecules and magnesium hydroxide, which fit well with the similar $\mathrm{pH}$ for all samples after the immersion. Comparing the intensities of different bands, it can be seen that the addition of BSA or FBS largely enhanced the band intensity from phosphates, which agrees with preceding

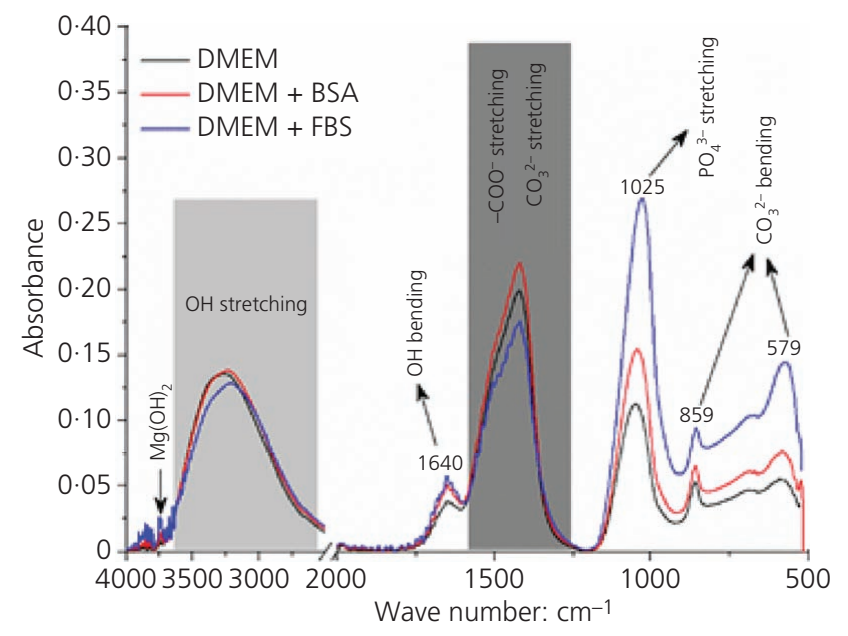

Figure 6. IR results for samples in DMEM-based media under semi-static conditions 
Table 1. Weight percentages of elements in the degradation products of samples under different conditions

\begin{tabular}{|c|c|c|c|c|c|c|c|}
\hline Conditions & Medium & Oxygen: wt. \% & Magnesium: wt. \% & Phosphorus: wt.\% & Carbon: wt. \% & Calcium: wt. \% & Sodium: wt. $\%$ \\
\hline \multirow[t]{3}{*}{ Static } & DMEM & 53.0 & $12 \cdot 7$ & $11 \cdot 1$ & $8 \cdot 2$ & $14 \cdot 1$ & 0.9 \\
\hline & DMEM + BSA & 54.6 & $16 \cdot 5$ & $9 \cdot 2$ & 8.7 & $9 \cdot 8$ & $1 \cdot 2$ \\
\hline & DMEM + FBS & $51 \cdot 8$ & $15 \cdot 2$ & $13 \cdot 2$ & $7 \cdot 8$ & $11 \cdot 2$ & 0.8 \\
\hline \multirow[t]{3}{*}{ Semi-static } & DMEM & $59 \cdot 8$ & $26 \cdot 2$ & 1.6 & $9 \cdot 0$ & $2 \cdot 5$ & 0.9 \\
\hline & DMEM + BSA & $54 \cdot 3$ & $16 \cdot 0$ & 11.4 & $8 \cdot 2$ & $9 \cdot 3$ & 0.8 \\
\hline & DMEM + FBS & $50 \cdot 6$ & $11 \cdot 2$ & $14 \cdot 3$ & $8 \cdot 2$ & $15 \cdot 0$ & 0.7 \\
\hline \multirow[t]{3}{*}{ Dynamic } & DMEM & $57 \cdot 2$ & $22 \cdot 7$ & $6 \cdot 3$ & $8 \cdot 7$ & $4 \cdot 1$ & 1.0 \\
\hline & DMEM + BSA & $52 \cdot 0$ & $14 \cdot 6$ & $12 \cdot 4$ & $8 \cdot 0$ & $12 \cdot 1$ & 0.9 \\
\hline & DMEM + FBS & $52 \cdot 7$ & $12 \cdot 9$ & $13 \cdot 1$ & $7 \cdot 7$ & $12 \cdot 9$ & 0.7 \\
\hline
\end{tabular}
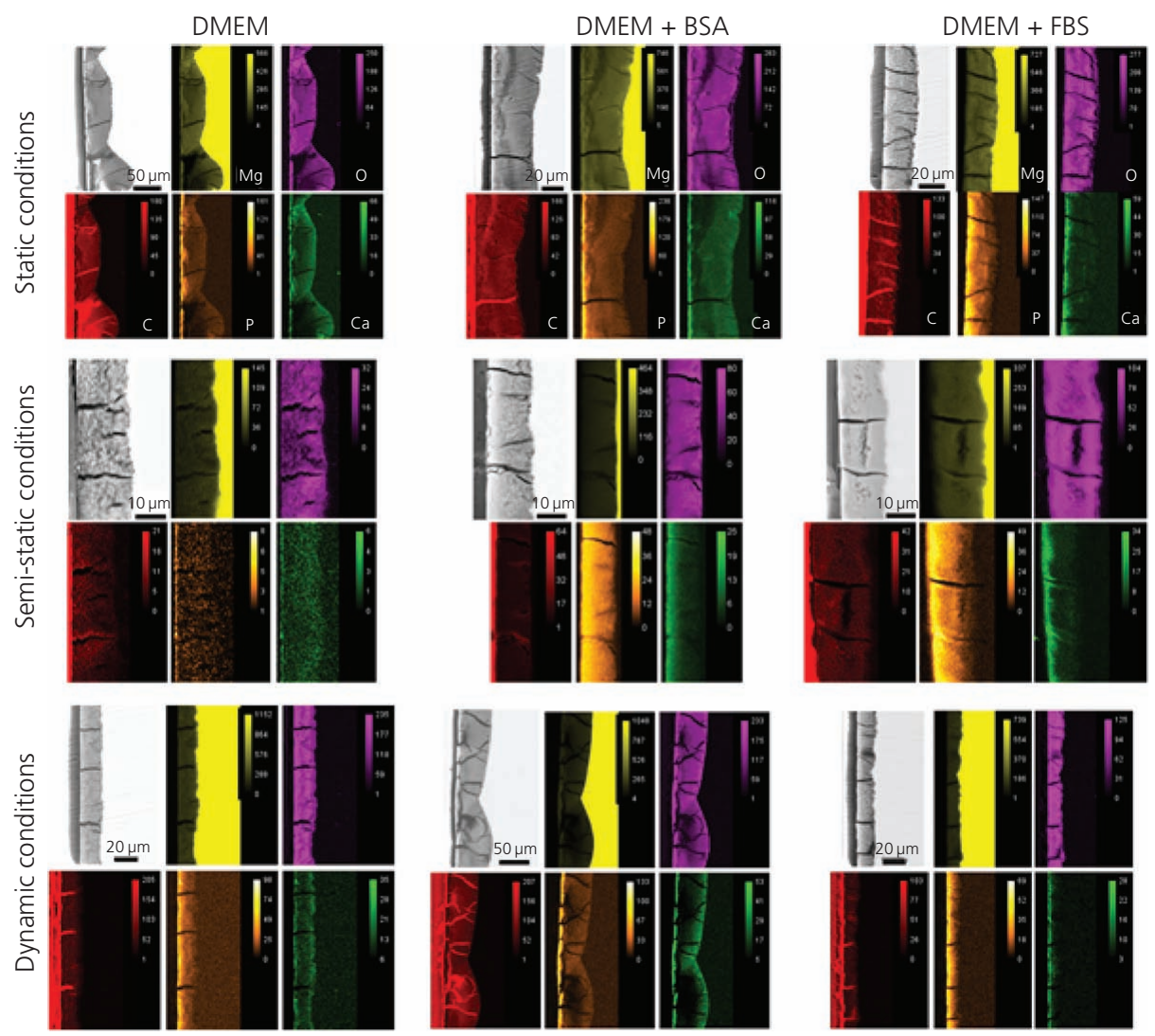

Figure 7. EDS element mapping of the representative cross-sectional degradation layer formed under different conditions

EDS result that proteins promote the formation of calcium-phosphorus products on the magnesium surface.

\subsection{Cross-sectional degradation morphology and layer elements}

To present the elemental distributions in the degradation layer, EDS mapping was conducted on the cross-sections of the degradation layer, as shown in Figure 7. Due to the low amount of sodium as listed in Table 1, it is not presented in Figure 7. The results showed that carbon was mainly distributed in resin, while oxygen and magnesium were abundant in the degradation layer. Calcium and phosphorus were mainly located in the top of the degradation layer, which results in the formation of a calcium/ phosphorus-rich top degradation layer no matter which condition or medium was used. Combined with IR results, this suggests the formation of calcium-phosphorus salts in the top of the magnesium surface. Furthermore, higher contents of phosphorus and calcium and a thicker top calcium/phosphorus-rich layer could be found in DMEM + BSA/FBS than in DMEM, particularly under semi-static and dynamic conditions. It should be noted that an obvious penetration of resin into the degradation layer could be found for the sample immersed in DMEM under static immersion and the sample in DMEM + BSA under the dynamic condition, indicating the breakage of the degradation layer, which is in agreement with the SEM observation (Figure 5). 


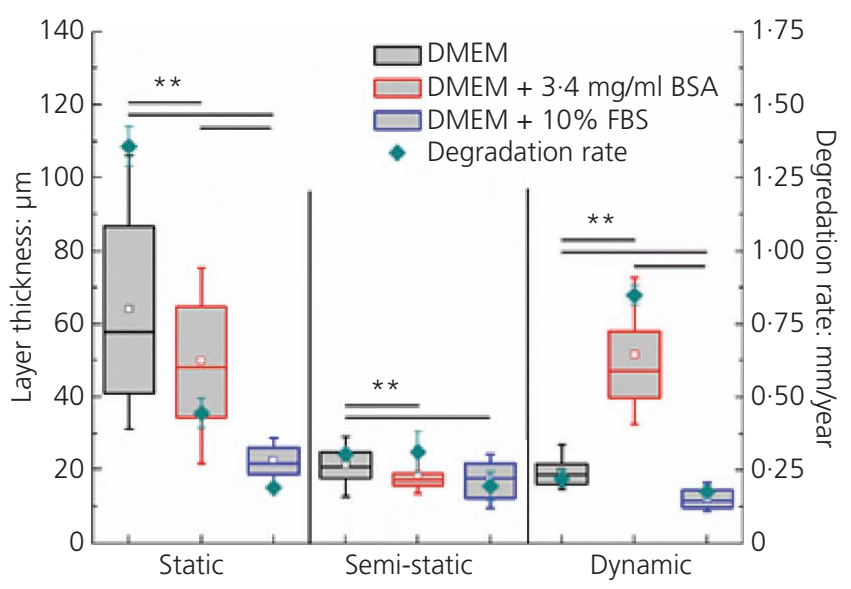

Figure 8. Thickness of the degradation layer formed in different media under different conditions (at least 80 points were taken) and the corresponding degradation rate of magnesium (The boxes (bottom to top) show the 25th percentile, median and 75th percentile. The whiskers mark the tenth and 90th percentiles; the rectangular points in the box are average values. Significance levels: ${ }^{*} p<0.05,{ }^{* *} p<0.01$ )

The thickness of the degradation layer in different media under different conditions was measured according to the cross-sections of the degradation layer, which is shown in Figure 8. It reveals the thickest degradation layer in DMEM under static immersion, while the thinnest one in DMEM + FBS under the dynamic condition, which fits well with the degradation rate of pure magnesium. The presence of BSA or FBS in the medium led to the formation of a thinner degradation layer, except for BSA under the dynamic condition. Comparing with the degradation rate of pure magnesium, it can be found that a higher degradation rate corresponds to a thicker degradation layer.

\section{Discussion}

To obtain more in vivo-like degradation, many researchers have always tried to control the $\mathrm{pH}$ of the testing medium within the physiological range $(7 \cdot 2-7 \cdot 4)$ by way of different buffering systems or medium-flow set-ups. ${ }^{11,36,37}$ In this study, a carbon dioxide/ bicarbonate system was used as the buffering system, and the $\mathrm{pH}$ of the medium under different conditions was in the range $7 \cdot 7-8 \cdot 3$ (Figure 4), indicating proper buffering used during immersion. Besides the buffering capacity, the introduction of carbon dioxide into the immersion system also affected the composition of degradation products, which promoted the formation of carbonate on the magnesium surface (Figure 6). This in turn contributed to the degradation rate and the $\mathrm{pH}$ increase. ${ }^{38,39}$ Moreover, the existence of calcium ions in the testing solution, combined with the presence of bicarbonate and hydrogen phosphate $\left(\mathrm{HPO}_{4}{ }^{2-}\right)$ ions, is proven to largely inhibit the increase in $\mathrm{pH}$ and the degradation of magnesium by way of the enhanced protective properties of degradation products on the surface. ${ }^{40,41}$ Thus, the carbon dioxide/ bicarbonate system and the proper medium used in this study guaranteed the suitable $\mathrm{pH}$ range for magnesium degradation during the test. All immersion tests, static, semi-static and dynamic, showed an initial increase in $\mathrm{pH}$ (Figure 4), which is a typical behavior of magnesium degradation due to the attack of fluid on the bare magnesium surface, which is in agreement with the report result. ${ }^{42}$ During the following immersion, the $\mathrm{pH}$ of the medium reached a relatively stable level (7·7-8.3) (Figure 4), except for the fluctuation of $\mathrm{pH}$ under the semi-static condition when the medium was refreshed. ${ }^{33,43}$ The general lower $\mathrm{pH}$ value in the medium containing proteins (except DMEM + BSA under the dynamic condition) was caused by not only the slower degradation rate of magnesium but also the buffering capacity of proteins (carboxyl and amino groups).

The results from the static immersion test showed the highest inhibition efficiency of proteins on magnesium degradation (Figure 3). When magnesium is immersed in the medium, magnesium dissolution occurs and leads to the release of magnesium ions $\left(\mathrm{Mg}^{2+}\right)$ and an increase in $\mathrm{pH}$. The reduction of oxygen is also possible for magnesium degradation as the cathodic reaction, leading to the less dissolved oxygen in the medium. ${ }^{44}$ Subsequently, depending on the product solubility, degradation products, such as magnesium/calcium- $\mathrm{PO}_{4}$, magnesium/calcium- $\mathrm{CO}_{3}$ and magnesium hydroxide, are formed on the surface (Figures 5 and 6), which in turn reduces the magnesium dissolution due to the barrier effect. In static immersion, the equilibrium between magnesium degradation and the formation of degradation products could be reached as the immersion is prolonged, as indicated by the stable degradation rate after long-term immersion. ${ }^{10,30}$ As reported in the literature, ${ }^{21,25,45}$ proteins can adsorb on the magnesium surface during immersion and then prevent the penetration of aggressive ions - for example, chloride ions $\left(\mathrm{Cl}^{-}\right)$. Thus, this can decrease the degradation rate of magnesium. On the other hand, the binding/chelating of proteins to magnesium ions can increase the degradation rate of magnesium due to its disturbance in the formation of degradation products. ${ }^{15,22,24,46}$ However, in this study, the addition of proteins in static immersion seemed to promote the formation of a calcium/phosphorus-rich top layer (Figure 7), which is believed to inhibit the degradation of magnesium. ${ }^{19}$ Since lower $\mathrm{pH}$ was determined in DMEM + BSA/ FBS than in DMEM (Figure 4), it can be stated that this promoted calcium-phosphorus salt formation was probably not caused by the supersaturation of calcium-phosphorus salts at higher $\mathrm{pH}$ but was induced by adsorbed proteins due to the attraction of organic groups to calcium/phosphate ions. ${ }^{20}$ Thus, the adsorption of proteins and the induced formation of calcium-phosphorus products play predominant roles in magnesium degradation in a protein-containing medium under static conditions; eventually the presence of proteins leads to slower degradation of magnesium.

Semi-static immersion has often been used to predict the degradation of magnesium in vivo, ${ }^{8,12,27}$ which is believed to be more 'realistic' than static immersion, since the immersion medium is refreshed after certain intervals. Ensuring that the same medium volume was used during immersion resulted in a lower 
$\mathrm{V} / \mathrm{S}$ for semi-static immersion $(1 \cdot 03)$ than for static condition $(5 \cdot 88)$, which easily led to the accumulation of magnesium and hydroxide $\left(\mathrm{OH}^{-}\right)$ions in the medium as reflected by the higher osmolality and slightly higher $\mathrm{pH}$ after $3 \mathrm{~d}$ of immersion (Figure 4). However, the degradation mechanism of pure magnesium was still the same as that under the static condition (Figure 5). When the medium was refreshed, the alteration of the environment surrounding the sample occurred suddenly, which repromoted the degradation of magnesium due to the lower $\mathrm{pH}$ and osmolality presented and the possible dissolution of precipitated products. Thus, the degradation of pure magnesium should be gradual under the semi-static condition as the medium was changed, while the degradation rate still showed a decreasing trend from the point of view of long-term immersion. ${ }^{30,42}$ In this case, protein adsorption had occurred on the magnesium surface before the medium change, which further impeded the penetration of the refreshed medium after the medium change, thereby leading to the inhibition of proteins on magnesium degradation (Figures 7 and 8). ${ }^{19}$ It should be noted that a lower inhibition efficiency of proteins was found under semi-static conditions compared with that under static immersion. This is a comprehensive result of many factors, such as $\mathrm{S} / \mathrm{V}$ ratio, testing duration and medium change intervals.

The introduction of flow into the immersion test enables investigation of the comprehensive effect of proteins and dynamic conditions on magnesium degradation. In general, fluid flow is believed to promote the degradation rate of magnesium due to the accelerated diffusion process and the induced shear stress. ${ }^{28,29,47}$ However, in this study, the degradation rate of pure magnesium in DMEM, DMEM + BSA and DMEM + FBS under dynamic conditions was slower than, faster than or similar to that under static immersion (Figure 2), suggesting the complex comprehensive effect of fluid flow and proteins on magnesium degradation. It has been reported that the flow condition promotes protein adsorption and seems to increase the extent of protein adsorption on the surface. ${ }^{48-50}$ However, all of these studies were performed on a stable (unchanged) surface, whereas magnesium presents an active variable surface during immersion. Thus, this leads to a more complex situation for protein adsorption on the magnesium surface under flow conditions, since fluid flow promotes not only the diffusion of ions/proteins in the medium from the medium to the degradation interface but also the diffusion of released ions (magnesium ions) from the degradation interface to the medium. Furthermore, their influence on the properties of the degradation layer, such as compactness and integrity, also plays a vital role in the degradation of magnesium (Figures 7 and 8). Therefore, protein adsorption behavior and protein performance in the medium under dynamic conditions needed to be studied further to obtain clearer understanding of the comprehensive influence of proteins and flow on magnesium degradation.

It is obvious that the inhibition efficiency of proteins on magnesium degradation (Figure 3), regardless of using BSA or
FBS, decreased from static and semi-static conditions to dynamic conditions, suggesting the deterioration of medium turbulence to the inhibition effect of proteins on magnesium degradation. Furthermore, in comparison with DMEM + BSA, a little change in the degradation rate $(0 \cdot 175-0.195 \mathrm{~mm} /$ year $)$ and degradation surface (Figures 2 and 7) was observed for DMEM $+10 \%$ FBS irrespective of the immersion condition used, demonstrating that the addition of FBS can weaken the difference in magnesium degradation caused by medium-flow conditions and present more comparable results for different conditions. These different effects for BSA and FBS are attributed to the complex composition of FBS, which contains transferrin, various growth factors and so on, compared with BSA. These results indicate a different effect of protein on magnesium degradation dependent on the implantation site - for example, a slow medium flow in cortical bone or muscle tissue suggests the inhibitive effect of BSA on magnesium degradation, while a relatively fast medium flow in blood vessels implies the accelerated influence of BSA on magnesium degradation. However, the interactions between proteins should be further taken into consideration when the effect of proteins on the degradation of magnesium under in vivo conditions is discussed.

\section{Conclusion}

In the present study, the comprehensive effects of proteins and flow conditions on the degradation of pure magnesium were investigated by using three different flow conditions, static, semistatic and dynamic conditions. The results revealed the inhibitive effect of protein to magnesium degradation under static conditions (decreased from $1.358 \pm 0.068$ to $0.444 \pm 0.052 \mathrm{~mm} /$ year for BSA and $0.188 \pm 0.001 \mathrm{~mm} /$ year for FBS) and the similar inhibition efficiency of FBS under semi-static and dynamic conditions, while BSA accelerated the degradation of magnesium from $0.301 \pm 0.017$ and $0.222 \pm 0.028$ to $0.309 \pm 0.074$ and $0 \cdot 849 \pm 0.035 \mathrm{~mm} /$ year under semi-static and dynamic conditions, respectively. This indicates that the decreased inhibition efficiency of proteins on magnesium degradation is in the order static > semi-static $>$ dynamic (decreased from $35 \cdot 7$ to -2.6 and $-281 \cdot 8 \%$ for BSA and from $87 \cdot 4$ to $35 \cdot 7$ and $19 \cdot 9 \%$ for FBS), demonstrating that the medium turbulence decreases the inhibition effect of protein on magnesium degradation. These results imply the flow-dependent influence of proteins on the degradation of magnesium-based implants considering the different flow conditions presented at different implantation sites - for example, fast flow in blood vessels but slow flow in cortical bone. To reduce the difference caused by flow during in vitro testing, a more physiological solution, DMEM + FBS, is recommended as the immersion medium for magnesium degradation due to the more comparable degradation surface (uniform degradation layer and element distribution in the degradation layer) and degradation rate (around $1.80 \mathrm{~mm} /$ year) under different conditions. Additionally, the bioreactor-based in vitro method facilitates the understanding of the comprehensive influence of proteins and flow on magnesium degradation under more physiological conditions, although more details about protein performance under flow need to be investigated. 


\section{Acknowledgements}

Dr. Ruiqing Hou would like to thank the financial support from the China Scholarship Council and Helmholtz Association of German Research Centers. The authors would like to acknowledge the help of Dr. Nico Scharnagl with IR measurements and the instruction from Jorge Gonzales for the dynamic tests.

\section{REFERENCES}

1. Zheng Y, Gu X and Witte F (2014) Biodegradable metals. Materials Science and Engineering: R: Reports 77: 1-34.

2. Zhao D, Witte F, Lu F et al. (2017) Current status on clinical applications of magnesium-based orthopaedic implants: a review from clinical translational perspective. Biomaterials 112: 287-302.

3. Kumar K, Gill R and Batra U (2018) Challenges and opportunities for biodegradable magnesium alloy implants. Materials Technology 33(2): 153-172.

4. Martinez Sanchez AH, Luthringer BJ, Feyerabend F and Willumeit R (2015) $\mathrm{Mg}$ and $\mathrm{Mg}$ alloys: how comparable are in vitro and in vivo corrosion rates? A review. Acta Biomaterialia 13: 16-31.

5. Myrissa A, Agha NA, Lu Y et al. (2016) In vitro and in vivo comparison of binary $\mathrm{Mg}$ alloys and pure $\mathrm{Mg}$. Materials Science and Engineering: $C$ 61: 865-874.

6. Marco I, Myrissa A, Martinelli E et al. (2017) In vivo and in vitro degradation comparison of pure $\mathrm{Mg}, \mathrm{Mg}-10 \mathrm{Gd}$ and $\mathrm{Mg}-2 \mathrm{Ag}$ : a short term study. European Cells \& Materials 33: 90-104.

7. Gonzalez J, Hou RQ, Nidadavolu EP, Willumeit-Römer R and Feyerabend F (2018) Magnesium degradation under physiological conditions - best practice. Bioactive Materials 3(2): 174-185.

8. Walker J, Shadanbaz S, Kirkland NT et al. (2012) Magnesium alloys: predicting in vivo corrosion with in vitro immersion testing. Journal of Biomedical Materials Research Part B: Applied Biomaterials 100(4): 1134-1141.

9. Yang L, Hort N, Willumeit R and Feyerabend F (2012) Effects of corrosion environment and proteins on magnesium corrosion. Corrosion Engineering, Science and Technology 47(5): 335-339.

10. Mei D, Lamaka SV, Gonzalez J et al. (2018) The role of individual components of simulated body fluid on the corrosion behavior of commercially pure Mg. Corrosion Science 147: 81-93.

11. Xin Y and Chu PK (2010) Influence of Tris in simulated body fluid on degradation behavior of pure magnesium. Materials Chemistry and Physics 124(1): 33-35.

12. Agha NA, Feyerabend F, Mihailova B et al. (2016) Magnesium degradation influenced by buffering salts in concentrations typical of in vitro and in vivo models. Materials Science and Engineering: $C$ 58: $817-825$.

13. Hou RQ, Scharnagl N, Feyerabend F and Willumeit-Römer R (2018) Exploring the effects of organic molecules on the degradation of magnesium under cell culture conditions. Corrosion Science 132: 35-45.

14. Wang Y, Ding BH, Gao SY et al. (2019) In vitro corrosion of pure $\mathrm{Mg}$ in phosphate buffer solution - influences of isoelectric point and molecular structure of amino acids. Materials Science and Engineering: C 105: article 110042.

15. Heakal FET and Bakry AM (2018) Serum albumin can influence magnesium alloy degradation in simulated blood plasma for cardiovascular stenting. Materials Chemistry and Physics 220: 35-49.

16. Mei D, Lamaka SV, Feiler C and Zheludkevich ML (2019) The effect of small-molecule bio-relevant organic components at low concentration on the corrosion of commercially pure $\mathrm{Mg}$ and $\mathrm{Mg}-0.8 \mathrm{Ca}$ alloy: an overall perspective. Corrosion Science 153: 258-271.

17. Liu D, Hu S, Yin X et al. (2018) Degradation mechanism of magnesium alloy stent under simulated human micro-stress environment. Materials Science and Engineering: C 84: 263-270.
18. Gao Y, Wang L, Li L et al. (2019) Effect of stress on corrosion of high-purity magnesium in vitro and in vivo. Acta Biomaterialia $\mathbf{8 3}$ : 477-486.

19. Hou RQ, Scharnagl N, Willumeit-Römer R and Feyerabend F (2019) Different effects of single protein vs. protein mixtures on magnesium degradation under cell culture conditions. Acta Biomaterialia 95 : 256-268.

20. Tavafoghi M and Cerruti M (2016) The role of amino acids in hydroxyapatite mineralization. Journal of the Royal Society Interface 13(123): article 20160462

21. Wagener $V$ and Virtanen S (2016) Protective layer formation on magnesium in cell culture medium. Materials Science and Engineering: C 63: 341-351.

22. Li T, He Y, Zhou J et al. (2018) Influence of albumin on in vitro degradation behavior of biodegradable $\mathrm{Mg}-1.5 \mathrm{Zn}-0.6 \mathrm{Zr}-0.2 \mathrm{Sc}$ alloy. Materials Letters 217: 227-230.

23. Willumeit R, Fischer J, Feyerabend F et al. (2011) Chemical surface alteration of biodegradable magnesium exposed to corrosion media. Acta Biomaterialia 7(6): 2704-2715.

24. Johnson I, Jiang W and Liu H (2017) The effects of serum proteins on magnesium alloy degradation in vitro. Scientific Reports 7(1): article 14335.

25. Hou RQ, Willumeit-Römer R, Garamus VM et al. (2018) Adsorption of proteins on degradable magnesium - which factors are relevant? ACS Applied Materials \& Interfaces 10(49): 42175-42185.

26. Gu X, Zheng Y and Chen L (2009) Influence of artificial biological fluid composition on the biocorrosion of potential orthopedic Mg-Ca, AZ31, AZ91 alloys. Biomedical Materials 4(6): article 065011.

27. Yamamoto A and Hiromoto S (2009) Effect of inorganic salts, amino acids and proteins on the degradation of pure magnesium in vitro. Materials Science and Engineering: C 29(5): 1559-1568.

28. Wang J, Jang Y, Wan G et al. (2016) Flow-induced corrosion of absorbable magnesium alloy: in-situ and real-time electrochemical study. Corrosion Science 104: 277-289.

29. Han L, Li X, Bai J et al. (2018) Effects of flow velocity and different corrosion media on the in vitro bio-corrosion behaviors of AZ31 magnesium alloy. Materials Chemistry and Physics 217: 300-307.

30. Dahms M, Höche D, Ahmad Agha N, Feyerabend F and Willumeit-Römer R (2018) A simple model for long-time degradation of magnesium under physiological conditions. Materials and Corrosion 69(2): 191-196.

31. Kieke M, Feyerabend F, Lemaitre J, Behrens $P$ and Willumeit-Römer R (2016) Degradation rates and products of pure magnesium exposed to different aqueous media under physiological conditions. BioNanoMaterials 17(3-4): 131-143.

32. Swain SK and Sarkar D (2013) Study of BSA protein adsorption/ release on hydroxyapatite nanoparticles. Applied Surface Science 286: 99-103.

33. Agha NA, Liu Z, Feyerabend F et al. (2018) The effect of osteoblasts on the surface oxidation processes of biodegradable $\mathrm{Mg}$ and $\mathrm{Mg}-\mathrm{Ag}$ alloys studied by synchrotron IR microspectroscopy. Materials Science and Engineering: C 91: 659-668.

34. Bowen PK, Drelich J and Goldman J (2014) Magnesium in the murine artery: probing the products of corrosion. Acta Biomaterialia 10(3): 1475-1483.

35. Jönsson M, Persson D and Thierry D (2007) Corrosion product formation during $\mathrm{NaCl}$ induced atmospheric corrosion of magnesium alloy AZ91D. Corrosion Science 49(3): 1540-1558.

36. Johnston S, Shi ZM and Atrens A (2015) The influence of $\mathrm{pH}$ on the corrosion rate of high-purity Mg, AZ91 and ZE41 in bicarbonate buffered Hanks' solution. Corrosion Science 101: 182-192.

37. Liu X, Yang H, Xiong P et al. (2019) Comparative studies of Tris- $\mathrm{HCl}$, HEPES and $\mathrm{NaHCO}_{3} / \mathrm{CO}_{2}$ buffer systems on the biodegradation behaviour of pure $\mathrm{Zn}$ in $\mathrm{NaCl}$ and $\mathrm{SBF}$ solutions. Corrosion Science 157: 205-219. 
38. Kirkland NT, Waterman J, Birbilis N et al. (2012) Buffer-regulated biocorrosion of pure magnesium. Journal of Materials Science: Materials in Medicine 23(2): 283-291.

39. Marco I, Feyerabend F, Willumeit-Römer R and Van der Biest $O$ (2015) Influence of testing environment on the degradation behavior of magnesium alloys for bioabsorbable implants. In TMS 2015 144th Annual Meeting \& Exhibition (The Minerals, Metals \& Materials Society (ed.)). Springer, Cham, Switzerland, pp. 497-506.

40. Mei D, Lamaka SV, Gonzalez J et al. (2019) The role of individual components of simulated body fluid on the corrosion behavior of commercially pure Mg. Corrosion Science 147: 81-93.

41. Jiang $P$, Blawert C, Scharnagl N and Zheludkevich ML (2019) Influence of water purity on the corrosion behavior of $\mathrm{Mg} 0.5 \mathrm{ZnX}(\mathrm{X}=$ $\mathrm{Ca}, \mathrm{Ge}$ ) alloys. Corrosion Science 153: 62-73.

42. Knigge SR and Glasmacher B (2018) Comparison between three in vitro methods to measure magnesium degradation and their suitability for predicting in vivo degradation. International Journal of Artificial Organs 41(11): 772-778.

43. Ahmad Agha N, Willumeit-Romer R, Laipple D, Luthringer B and Feyerabend $F$ (2016) The degradation interface of magnesium based alloys in direct contact with human primary osteoblast cells. PLoS One 11(6): article e0157874.
44. Silva EL, Lamaka SV, Mei D and Zheludkevich ML (2018) The reduction of dissolved oxygen during magnesium corrosion. ChemistryOpen $\mathbf{7 ( 8 )}$ : 664-668.

45. Zhang J, Kong $N$, Shi $Y$ et al. (2014) Influence of proteins and cells on in vitro corrosion of $\mathrm{Mg}-\mathrm{Nd}-\mathrm{Zn}-\mathrm{Zr}$ alloy. Corrosion Science 85: 477-481.

46. Harandi SE, Banerjee PC, Easton CD and Raman RS (2017) Influence of bovine serum albumin in Hanks' solution on the corrosion and stress corrosion cracking of a magnesium alloy. Materials Science and Engineering: C 80: 335-345.

47. Koo Y, Lee HB, Dong Z et al. (2017) The effects of static and dynamic loading on biodegradable magnesium pins in vitro and in vivo. Scientific Reports 7(1): article 14710.

48. Yu K, Andruschak P, Yeh HH, Grecov D and Kizhakkedathu JN (2018) Influence of dynamic flow conditions on adsorbed plasma protein corona and surface-induced thrombus generation on antifouling brushes. Biomaterials 166: 79-95.

49. Salim M, O'Sullivan B, McArthur SL and Wright PC (2007) Characterization of fibrinogen adsorption onto glass microcapillary surfaces by ELISA. Lab Chip 7(1): 64-70.

50. Choi EJ, Foster MD, Daly S et al. (2003) Effect of flow on human serum albumin adsorption to self-assembled monolayers of varying packing density. Langmuir 19(13): 5464-5474.

\section{How can you contribute?}

To discuss this paper, please submit up to 500 words to the journal office at journals@ice.org.uk. Your contribution will be forwarded to the author(s) for a reply and, if considered appropriate by the editor-in-chief, it will be published as a discussion in a future issue of the journal.

ICE Science journals rely entirely on contributions from the field of materials science and engineering. Information about how to submit your paper online is available at www.icevirtuallibrary.com/page/authors, where you will also find detailed author guidelines. 